\title{
Design and Application of a Magnetoelectric Composite Sensor for Pipeline Defect Detection
}

\author{
Haibo Zhu ${ }^{\mathrm{a}}$, Huadong Song ${ }^{\mathrm{a}, 1}$, Xiaoting Guo ${ }^{\mathrm{a}}$, Wenqiang Zhang ${ }^{\mathrm{a}}$, \\ Yunpeng Song ${ }^{\mathrm{a}}$, Chunfeng $\mathrm{Xu}^{\mathrm{a}}$, Yueting $\mathrm{Yu}^{\mathrm{a}}$ \\ a Shenyang Academy of Instrumentation Science Co., Ltd, Shenyang, 110043, China
}

\begin{abstract}
Nowadays, there are 160000 kilometers of pipelines in China. How to get more pipeline information in pipeline integrity management is a vitally important research topic. This paper designs a magnetoelectric composite sensor which is suitable for the non-destructive detection of the buried pipeline. according to the defect characteristics. this paper introduces the working principle, structure and characteristics of the composite sensor. Combined with the advantages of the magnetic sensor which is sensitive to the volume defects of the tube wall and the high frequency eddy current sensor which is sensitive to the near-surface defects of the tube wall on the principle of magnetic flux leakage, it realizes the identification and evaluation of the inner and outer wall defects of the tube wall. In this paper, the pipeline in the traction test field is taken as the test object. The test results show that there are 37 defects picked up by the magnetic sensor and 19 defects judged by the eddy current sensor.
\end{abstract}

Keywords. Sensor, eddy current, defect of pipeline, defect recognition, pull testing

\section{Introduction}

The pipeline is known as the national energy artery, which vital important to the national energy supply. With the increase of the service life of the pipeline, due to the role of corrosion and stress, various types of defects will appear in the pipeline. Therefore, it is necessary to detect the pipeline regularly and effectively, eliminate the defects in time, and reduce the occurrence of pipeline rupture accidents.

At present, various nondestructive testing systems have been proposed, such as the Conventional ultrasonic testing system, electromagnetic ultrasonic testing system, eddy current testing system and magnetic flux leakage detection systems ${ }^{[1-2]}$. The existing detection systems and detection probes can not meet the existing detection requirements duo to the complex structural characteristics and various forms of defects of the inner wall of the pipeline. Therefore, how to develop a new detection system to solve the above problems has become an urgent problem. This paper introduces the principle, application conditions and advantages of MFL testing and eddy current testing, and designs a

\footnotetext{
${ }^{1}$ Corresponding Author: Huadong Song, professor of engineering. The main research direction is nondestructive testing. E-mail: huadong_song800713@126.com.
} 
magnetoelectric composite sensor for nondestructive testing of oil and gas pipelines. The probe is used in the dragging test of the detector in the pipeline, and the test data show that it can not only detect the pipeline defects, but also distinguish the inner wall defects and the outer wall defects, which has far-reaching significance for the subsequent engineering application of the probe.

\section{Detection principle and characteristics of magnetoelectric composite sensor}

The principle of MFL detection is to use the high permeability of ferromagnetic materials to measure the change of permeability in ferromagnetic materials due to the existence of defects $^{[3-4]}$. The principle of pipeline magnetic flux leakage detection is shown in Figure 1. The two parts of the pipe wall are the longitudinal section view of the pipe. The upper pipe wall has a defective bottom wall. During the detection process, the permanent magnet magnetizes the pipe to be tested. When the tube wall is complete, defect-free and the material of the tube wall is uniform, all the magnetic lines pass through the magnetic circuit composed of the tube wall, permanent magnet, wire brush and soft iron. When the tube wall reaches the saturation magnetization state, if the tube wall is defective, part of the magnetic flux passing through the defect will still pass through the magnetic circuit, but a small amount of magnetic lines will be twisted at the defect, and leak to the outside of the tube wall along the edge of the defect So as to form magnetic flux leakage in the surrounding air. When the sensor passes through the defect free pipe wall, the output voltage does not change, because there is no magnetic flux leakage change. When the defect pipe wall passes, the voltage change achieves the purpose of defect detection ${ }^{[5-6]}$. The analysis of magnetic flux leakage signal can accurately judge the defect.

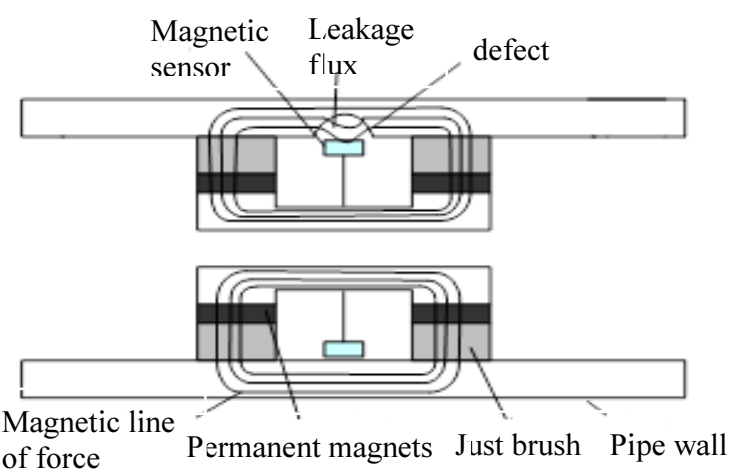

Figure 1. Schematic diagram of pipeline magnetic flux leakage detection

The application of magnetic sensor in magnetic flux leakage detection system has high corrosion sensitivity, which is suitable for high temperature, cold, underwater and other harsh environments. However, the magnetic sensor can only detect the volume defects of axial length, circumferential width and radial depth of the wall of the pipeline. In addition, even if the magnetic sensor can detect the defects in the pipe, it can not distinguish whether the defect locates at the inner wall or the outer wall.

According to the electromagnetic induction law, when the magnetic flux in the closed metal conductor changes, the closed induction eddy current will be generated in the 
conductor, which will hinder the change of the magnetic flux ${ }^{[7-8]}$. The working principle of the eddy current displacement sensor is shown in Figure 2.

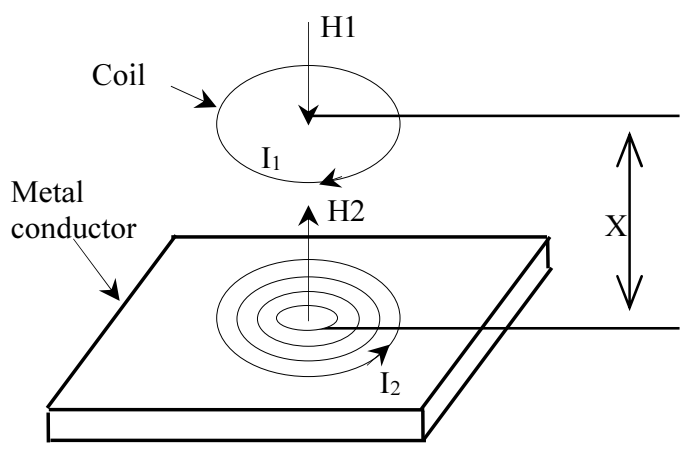

Figure 2. Working principle of eddy current displacement sensor

The induction coil is excited by the alternating current signal, which produces Joule heat and hysteresis loss, which causes the loss of alternating magnetic field energy and changes the equivalent impedance $Z$ of the sensor. The factors affecting the impedance $Z$ include the conductivity of the measured conductor $\sigma$, permeability $\mu$, excitation frequency $\mathrm{f}$ of the coil, displacement $\mathrm{X}$ between the sensor and the measured conductor, as long as these factors only change the displacement $X$, If other factors do not change, the equivalent impedance $\mathrm{Z}$ of the sensor will become a single function $\mathrm{Z}$ (x) of displacement $\mathrm{x}$, and the change of $\mathrm{Z}$ can reflect the change of $\mathrm{X}$, so as to realize the purpose of measuring displacement $\mathrm{X}$ after linearization.

Eddy current testing system is a non-destructive testing method based on the principle of electromagnetic induction. It has the advantages of fast response, high sensitivity, non-contact and no need for coupling medium. Therefore, it is especially suitable for the detection of inner wall defects of metal pipes ${ }^{[9-10]}$. However, the sensors in the traditional eddy current testing system generally have the problems of poor consistency, easy detection of the signal, hard to achieve high detection efficiency and high resolution simultaneously, and the poor adaptability to the detection object ${ }^{[11-12]}$.

\section{System implementation of magnetoelectric composite sensor}

The digital three-axis magnetic sensor transmits the collected leakage magnetic field to the ARM-STM32 control system of the probe through the IIC protocol. At the same time, the eddy current sensor will keep generating eddy current signal. The returned signal will be received by the eddy current coil receiving circuit and transmitted to the ARM-STM32 control probe system via the SPI protocol. It is transmitted to the data acquisition system $1.5 \mathrm{~m}$ away via the data output driver after being collected and stored by the control system. 


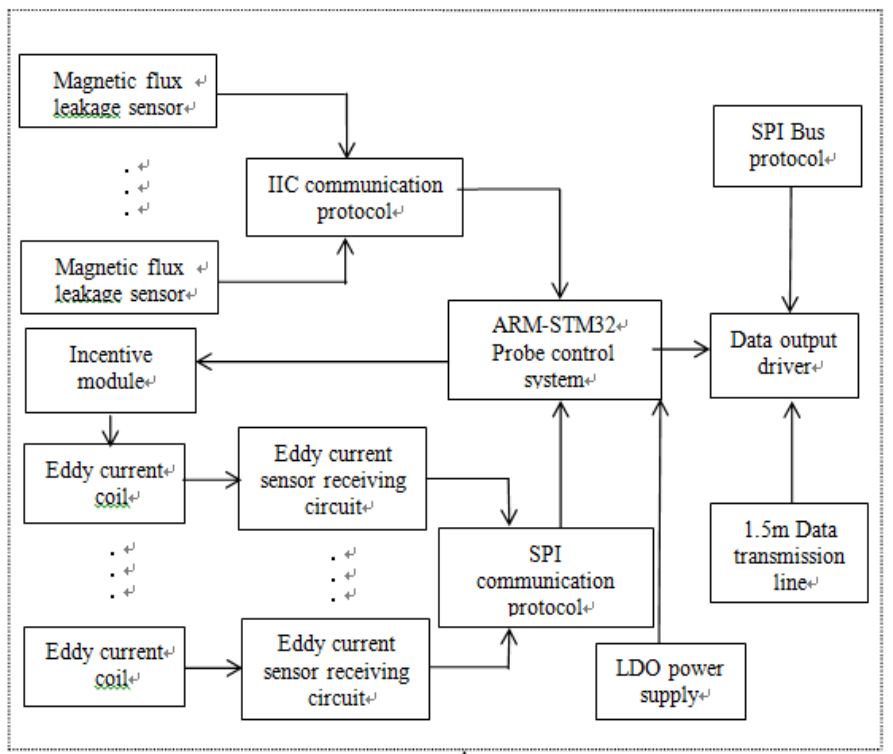

Figure 3. Composite sensor structure block diagram

Using PCB printed eddy current sensor coil instead of the traditional manual winding coil reduces coil volume and improves coil stability and uniformity.

After the eddy current sensor is excited, the eddy current signal generated is collected by the receiving circuit, and the receiving circuit diagram is shown in Figure 4.

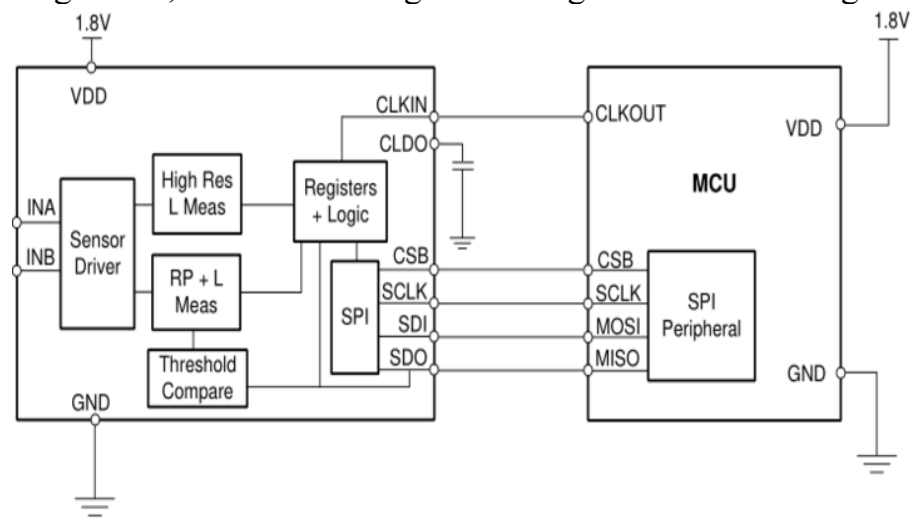

Figure 4. Eddy current sensor coil receiving circuit structure block diagram

The two ends of the eddy current sensor are connected with the signal terminals INA and INB of the LC oscillation circuit. The LC circuit provides excitation voltage, excitation frequency and other relevant parameters during the working process of the eddy current sensor. When the eddy current sensor coil detects a defect in the inner tube wall, its impedance and inductive reactance will change. They are detected by the inductance measurement circuit. Once the inductance value is processed by the register and logic processor, the threshold value is compared and converted into the output signal of the SPI communication protocol to identify the defects of the pipe wall.

As shown in Figure 4, the CSB, SCLK, SDI and SDO signals output by the SPI communication protocol are transmitted to the MCU acquisition system through the 
transmission line. The MCU host selects ARM-STM32 series chips, where CLKIN represents the external time base clock input, CLDO connects $15 \mathrm{nf}$ capacitor from pin to GND, CSB indicates the start of SPI transmission protocol, SCLK represents the SPI communication protocol clock input, SDI indicates SPI data input Connected to the MOSI of the SPI master, SDO indicates that the SPI data output is connected to the MISO of the SPI master.

Sensor detection data is transmitted to ARM-STM32 control probe system through the SPI protocol. The system includes a high-precision clock module, a stabilized voltage power module, a memory module, a bus protocol module and a FATFS file management module display module for collecting and storing sensor data to display the current output signal.

\section{Traction test results and analysis}

The magnetoelectric composite sensor is installed on the inner detector of the pipeline, which is used to detect the defects of the pipeline and distinguish whether they are inner wall defects or outer wall defects.

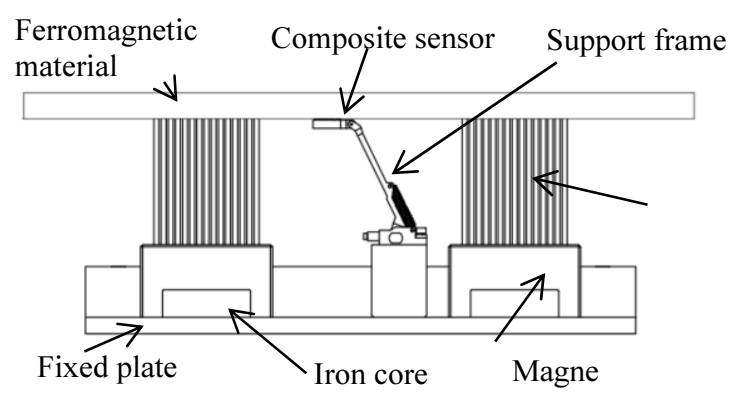

Figure 5. Magnetoelectric composite sensor detection device

In order to verify the reliability and functional integrity of the sensor, the pull test of the corresponding structure is carried out, and the state diagram of the structure before and after the pull test is shown in Figure 6.
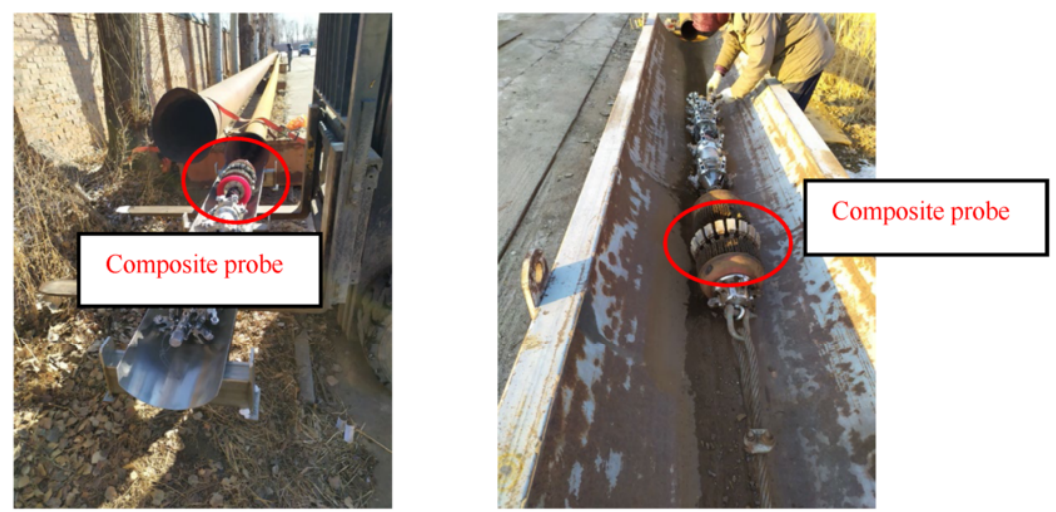

Figure 6. State degree of equipment traction test 
The detection results of the digital three-axis magnetic sensor of the composite probe are shown in Figure 7.

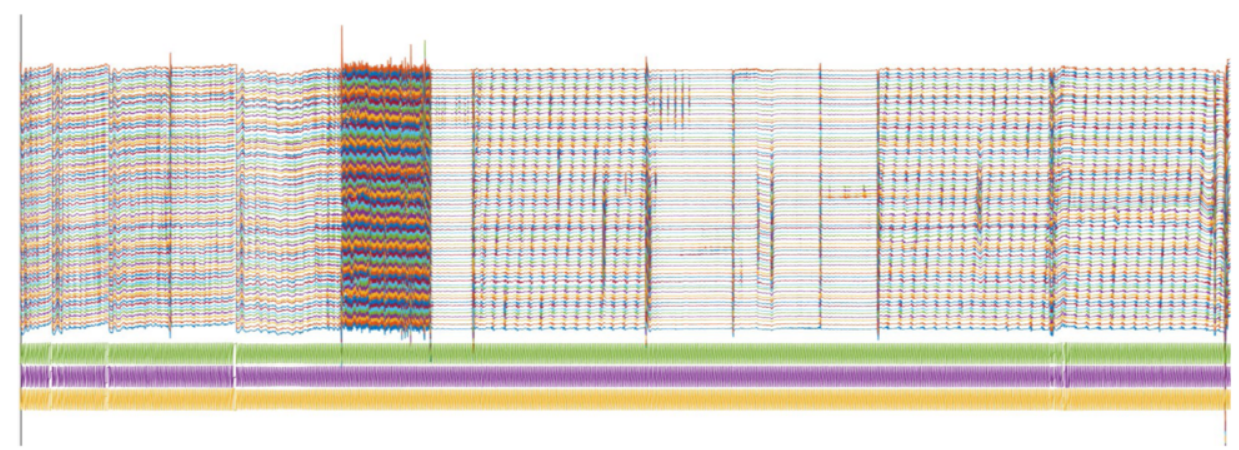

Figure 7a. Z-axis test data of composite probe

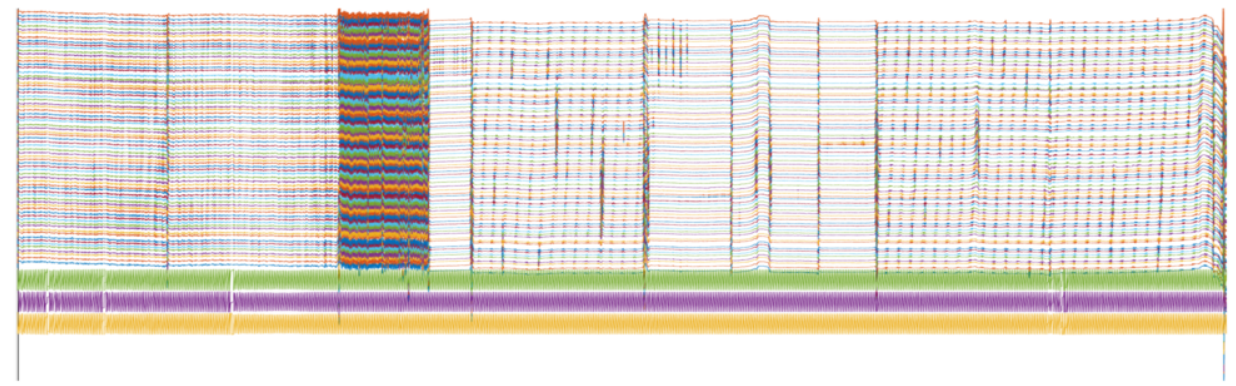

Figure 7b. Y-axis test data of composite probe

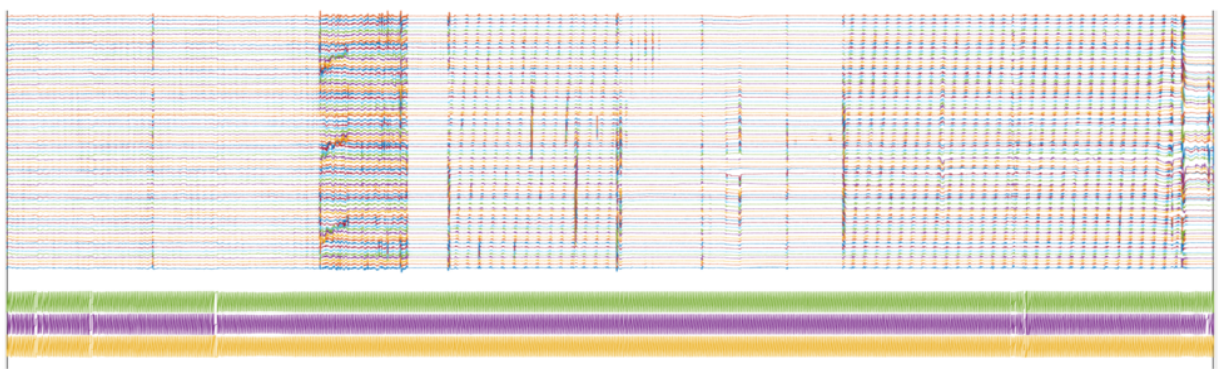

Figure 7c. X-axis test data of composite probe

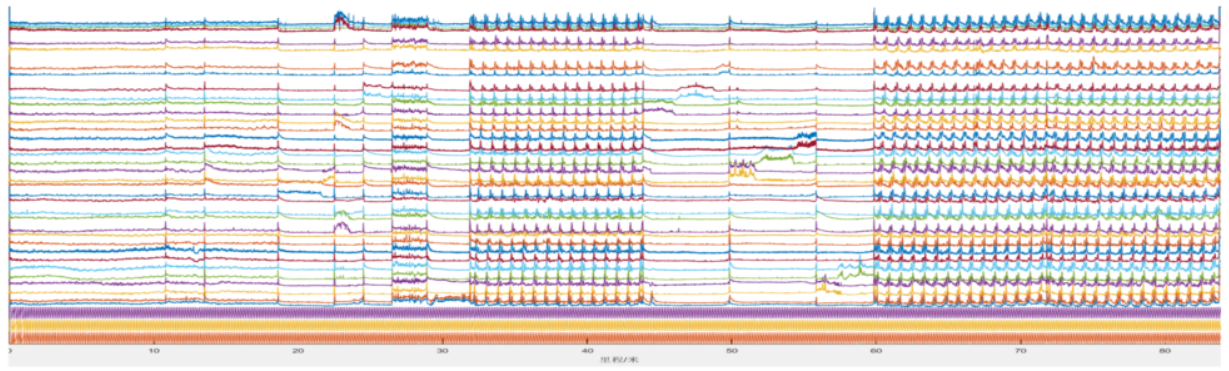

Figure 7d. Composite probe inductive reactance signal 


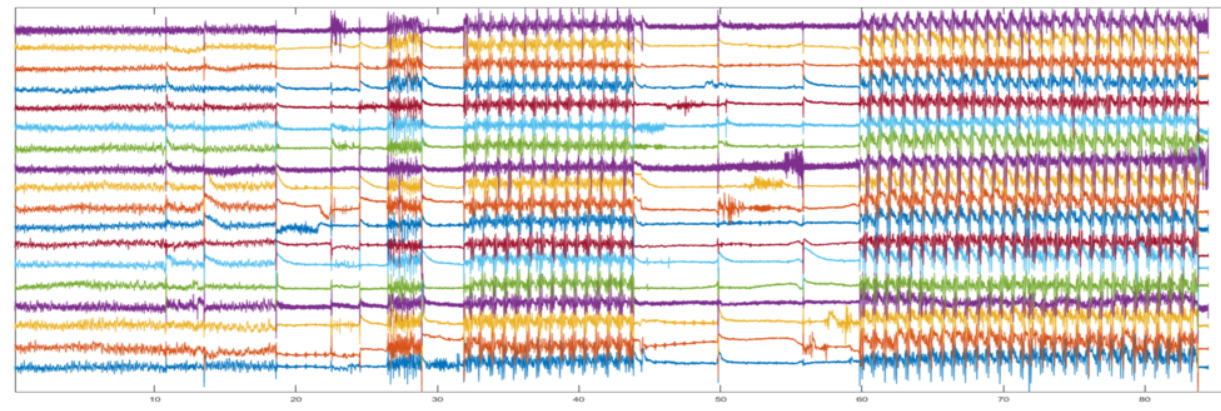

Figure 7f. Composite probe impedance signal

Figure 7. Composite sensor detection defect results

Through Figure 7, it can be seen the signals collected by the composite probe in the traction test pipeline. a, b and c display the data collected by the digital three-axial magnetic sensor, $\mathrm{d}$ and $\mathrm{f}$ display the impedance and inductive reactance signals collected by the eddy current sensor. Through comparison, it can be seen that the inspection data of $\mathrm{Z}$-axis and $\mathrm{Y}$-axis of composite probe are obvious, and the inspection data of X-axis can only be used as the supplement of the inspection data of other directions; the eddy current sensor is more sensitive to the straight weld and girth weld of pipeline, as well as some defects.

The function of the composite probe is mainly to distinguish the internal and external wall positions of the pipeline defects. According to Figure 7, it can be seen that the data of a certain section of the pipeline is relatively messy. This section is actually a seamless steel pipe laying, and a large number of characteristic defects are artificially designed. The following article mainly analyzes the detection data of the composite probe in this section. The data shown in figure8 (Because there are many defects that can not be pointed out completely, the red circle position is a relatively obvious defect).

It can be seen from Figure. 8 that the pipe section with the disorderly signal is laid by two seamless steel pipes. The seamless steel pipe has a certain influence on the detection of the magnetic sensor, and its data fluctuates greatly, but the existence of pipe defects can also be analyzed. According to the construction data, the defects of the front section are all the inner wall, and the defects of the rear section are all the outer wall. According to the detection of the magnetic sensor, the inner and outer walls of defects can not be distinguished in three directions. However, eddy current sensor detection shows that no matter the inductive reactance signal or impedance signal, it will not be affected by the straight seam steel pipe. The front section of the pipe shows defects, and the rear section shows no defects.

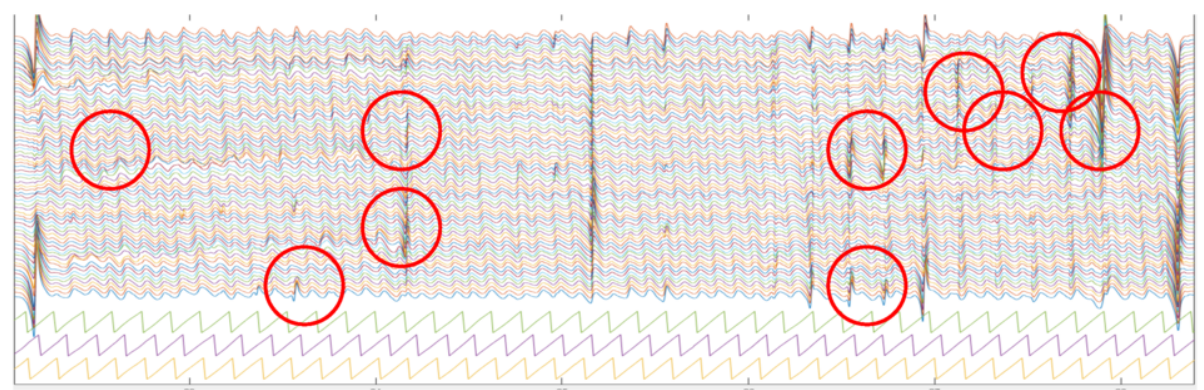

Figure 8a. Z-axis test data of composite probe 


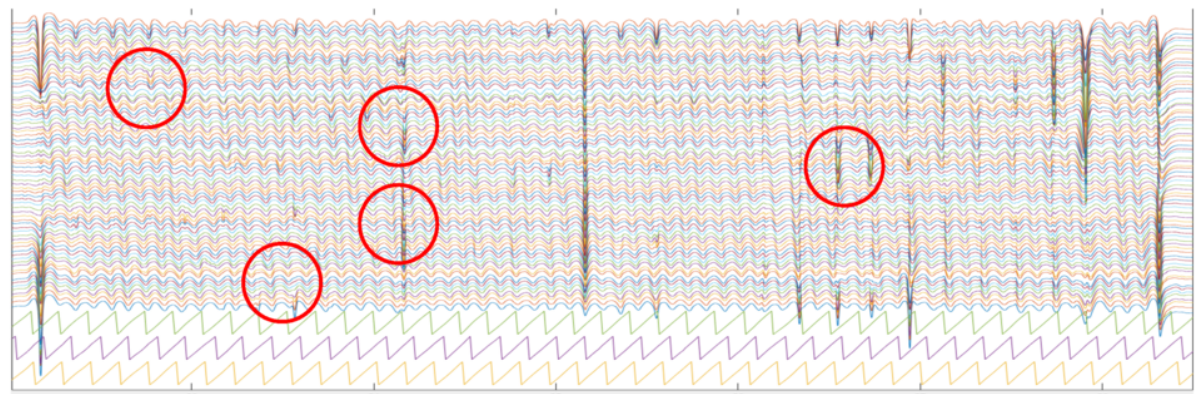

Figure 8b. Y-axis test data of composite probe

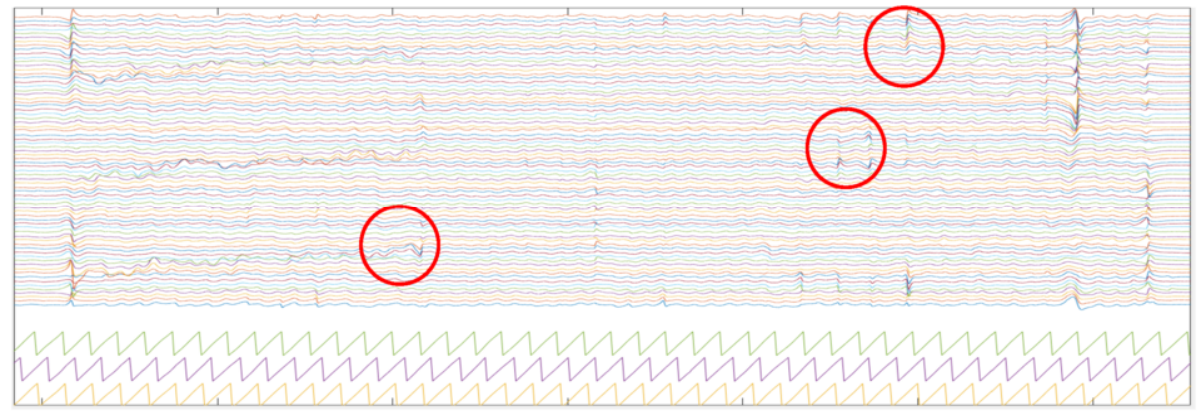

Figure 8c. X-axis test data of composite probe

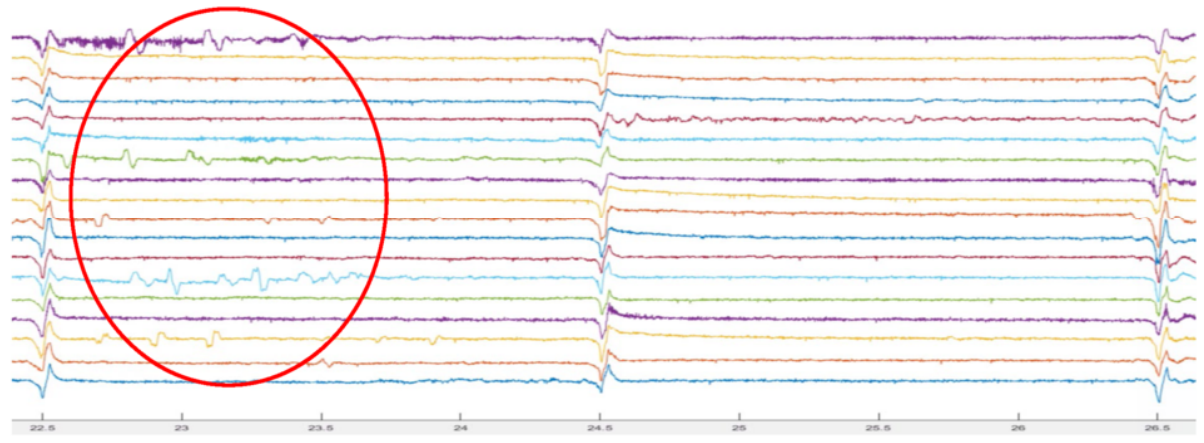

Figure 8d. Composite probe inductive reactance signal

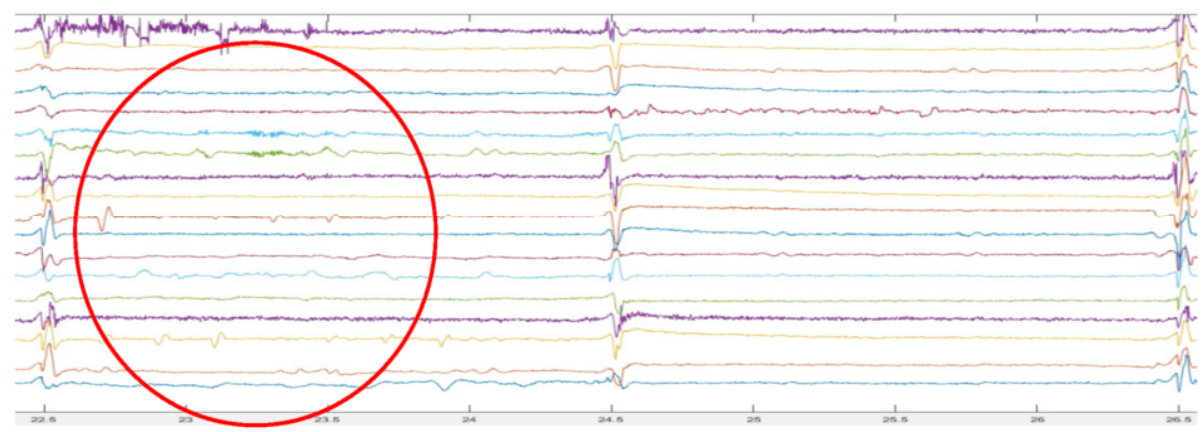

Figure 8f. Composite probe impedance signal

Figure 8. Composite sensor detection defect results 


\section{Conclusion}

In this paper, a composite sensor system for the detector in the pipeline is designed and developed. The principle, structure and function of the system are introduced in detail. The practicability and function of the probe are verified by pull test. Traction test results show that:

1. Both digital three axial magnetic sensor and eddy current sensor can detect the girth weld and spiral weld of pipeline.

2. The composite probe can not only detect the defects of the pipeline, but also distinguish the inner and outer walls of the pipeline defects.

3. For seamless steel pipes, eddy current testing has better stability and can supplement the magnetic signal very well.

\section{References}

[1] Xu Wei, Huang Song-ling, Zhao Wei, Xu Peng, Method and Implementation of Oil and Gas Pipeline Deformation Detection Based on Low Frequency Eddy Current, Electrical Measurement and Instrumentation 47 (2010), 10-15.

[2] Bao Qing-jun, Shuai Jian, Research Progress in Detection Technology of Oil and Gas Pipelines, Journal of Modern Chemical Industry 46(2017), 298-301.

[3] Huang K, 3-D Defect Profile Reconstruction from Magnetic Flux Leakage Signatures Using Wavelet Basis Function Neural Networks, Ames IA: Iowa State University, 2000.

[4] Huang Song-ling, et al, Theory and Application of Magnetic Flux Leakage Detection in Oil and Gas Pipelines, Beijing: Mechanical Industry Press 9(2013), 25-26.

[5] Paulsen J.A, Jiles, A Magnetic Imaging System for Evaluation of Material Conditions Using Magnetoresistive Devices, I-EEE Tansactions on Magnetics 39(2003), 3453-3455.

[6] Wang Fu-xiang, Feng Qing-shan, Zhang Hai-liang, Song Han-cheng, Chen Jian, Pipeline Feature Recognition Based on Three-axis Magnetic Flux Leakage Detection Technology, Non-destructive Testing 33(2011), 79-84.

[7] Xu Lin, Wang Heng, Huang Wei, Li Boquan et al, Simulation of Eddy Current Sensor Based on COMSOL Finite Element Method, Journal of Drainage and Irrigation Machinery Engineering 12(2015), 1097-1104.

[8] Yan Yan-chun, Zhu Xiao-zhi, Discussion on Performance Test Requirements of Eddy Current Testing System Based on NB/T47013-2015, Non-destructive Testing 39(2017), 69 72.

[9] Dong Shao-hua, Ji Shou-hong, Zhang Wei-wen, Zhao Xiaoli et al, Development and Industrial Application of G4 High-definition Composite Detector, Pipeline Protection 1 (2018).

[10] Ding Zhan-wu, He Ren-yang, Liu Zhong, Simulation Analysis and Quantization Model of Defect Signals in Pipeline Magnetic Flux Leakage Detection, Non-destructive Testing 35(2013), 30-33.

[11] Ye Ling-wei, Feng Wei. Overview of Eddy Current Testing Research, Fluid Dynamics 6(2018), 1-4.

[12] Xiao Chun-yan, Zhu Heng-jun, Qi Hong-yuan et al, Electromagnetic Detection Model of Ferromagnetic Material Hardness, Journal of Metrology 23(2002), 294-298. 